\title{
Using an Evolutionary Algorithm for the Tuning of a Chess Evaluation Function Based on a Dynamic Boundary Strategy
}

\author{
Hallam Nasreddine and Hendra Suhanto Poh \\ Faculty of Engineering and Computer Science \\ The University of Nottingham, Malaysia Campus \\ Semenyih, Malaysia \\ kcznh@nottingham.edu.my, hendrasutanto@gmail.com
}

\begin{abstract}
One of the effective ways of optimising the evaluation function of a chess game is by tuning each of its parameters. Recently, evolutionary algorithms have become an appropriate choice as optimisers.
\end{abstract}

In the past works related to this domain, the values of the parameters are within a fixed boundary which means that no matter how the recombination and mutation operators are applied, the value of a given parameter cannot go beyond its corresponding interval.

In this paper, we propose a new strategy called "dynamic boundary strategy" where the boundaries of the interval of each parameter are dynamic. A real-coded evolutionary algorithm that incorporates this strategy and uses the polynomial mutation as its main exploitative tool is implemented.

The effectiveness of the proposed strategy is tested by competing our program against a popular commercial chess software. Our chess program has shown an autonomous improvement in performance after learning for hundreds of generations.

Keywords - evaluation function, evolutionary algorithm, chess program.

\section{INTRODUCTION}

Chess, or "The Game of the Kings", is a classic strategy board game involving two players. It consists of 32 chess pieces and an $8 \times 8$ squares board, where the goal of the game is to capture the opponent's king. The first chess playing machine was built in 1769. Amazingly, the first ever chess program was written before computers were invented and this has gone onto become one of the popular research areas in computational intelligence. Since then, researchers have been trying to find a solution that allows the machine to play a perfect chess game.

In order to achieve this, the machine is required to traverse the chess game's search tree up to depth 100 (where each player is allowed to move no more than fifty times in a game). This is simply computationally impossible, as the average of possible moves (branching factor) per turn is around 35 moves.

\author{
Graham Kendall \\ School of Computer Science and IT \\ The University of Nottingham, Jubilee Campus \\ Nottingham, UK \\ gxk@cs.nott.ac.uk
}

Searching all the way down to the bottom of the search tree is still computationally impossible even after using pruning techniques (such as alpha-beta pruning) to cut off unnecessary branches of the tree. Therefore, practitioners would normally limit the search depth and apply some evaluation function to estimate the score of a given move. The setup of such an evaluation function can be done by iteratively tuning it until it reaches an optimum state. The search space of the possible values of the evaluation function, being so large, suggests the use of evolutionary algorithms might be applicable.

In this work, we use an evolutionary algorithm to search for a good evaluation function. Using the mutation operator and applying it on a dynamic interval, we try to evolve an adequate weight (denoting the importance) for each chess piece.

This paper is organised as follows. Section 2 briefly presents the implemented chess program. Section 3 describes the key modelling concepts of our application. The chromosomal representation, the selection method, and the mutation technique are all discussed. In particular, the dynamic boundary strategy is explained. Experimental designs and their results are discussed in section 4. Finally, section 5 gives the concluding remarks of this work.

\section{The Chess ENGINE}

A chess program is implemented to support our experiments. The positions of the chessboard are represented by a matrix of size $8 \times 8$, where each of its variables corresponds to a square on the chessboard. The basic rules of the chess game are implemented in the chess program to prevent illegal moves.

The chess program determines each move by evaluating the quality of each possible board position using an evaluation function. The evaluation function used in this work is a slight modification of the one used in [1] which is a simplified version of Shannon's evaluation function [5]. 
The evaluation function, given below in (1), calculates the sum of the material values a) for each chess piece, b) the number of two pawns existing on the same column (double pawn) and c) the number of available legal moves (mobility). Different weights are assigned to each of the chess pieces, double pawn, and mobility variables. These weights are to be tuned later by a learning process in order to optimise the evaluation function.

$$
\text { Eval }=\sum_{i=0}^{7} w[i]\left(Q[i]_{\text {White }}-Q[i]_{\text {Black }}\right)
$$

Where:

$\mathrm{Q}, \mathrm{W}=$ Quantity, Weight.

$\mathrm{Q}[7]=\{\mathrm{Q}$ king, $\mathrm{Q}$ queen, $\mathrm{Q}$ rook, $\mathrm{Q}$ bishop, $\mathrm{Q}$ knight, $\mathrm{Q}$ pawn, Q double pawn, Q mobility\}

$\mathrm{W}[7]=\{\mathrm{W}$ king, $\mathrm{W}$ queen, $\mathrm{W}$ rook, $\mathrm{W}$ bishop, $\mathrm{W}$ knight, W pawn, W double pawn, $\mathrm{W}$ mobility

The alpha-beta pruning procedure detailed in [9] is used to search for the best move from the chess game tree. The depth of the search is set to 3 ply.

In addition, for each chess piece captured and before the move is committed, a quiescence check operation is carried out. This process provides another 3 ply search.

The game is terminated when one of the three conditions is fulfilled: a checkmate state, no legal moves for a given player, or both players have reached 50 moves without winning. A player receives 1 point for a win and 0 for a draw or a loss.

\section{METHOD}

We use an evolutionary algorithm to optimise the evaluation function. An individual is represented by a real vector of dimension 6. Each vector element represents the weight of either a chess piece other than the king and the pawn (which are assigned constant weights of 1000 and 1 respectively), a double pawn, or a mobility.

There are five individuals in any given population. They all compete with each other for survival. Two individuals (two chess programs using two different evaluation functions) play each other in a two round game where each one will take turns in starting the game first. If an individual wins, it is awarded +1 , otherwise 0 . Based on the points collected from this tworound game, the winner is cloned and its clone mutated. Both the winner and its mutated version are copied into the next generation. This process continues until all individuals in the population converge to the same solution.

\section{A. Selection}

A vector population of five individuals is used where the selection process, a slight modification of the one in [1], is as follows. First we choose the first individual and make it compete with a randomly chosen individual from among the rest of the population.

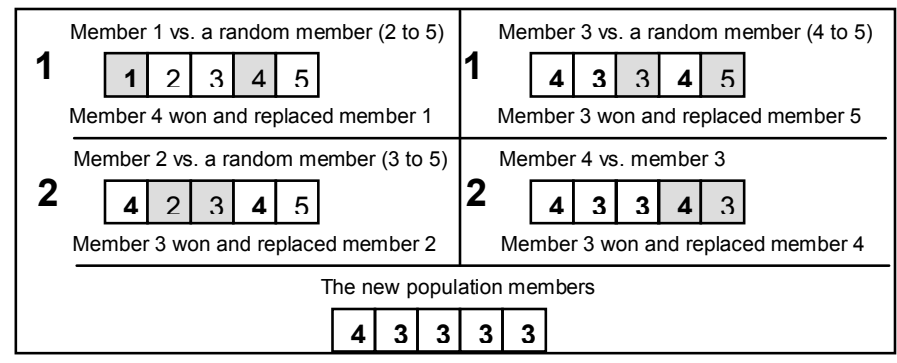

Figure 1. An Example of Selection-Competition

The winner is duplicated and overwrites the loser. Next, the second individual is selected and competes with a randomly chosen individual from the rest of the population excluding the first individual. This iterative procedure goes on until all the individuals have been considered. Figure 1 shows a complete selection of a vector population of five individuals. The shaded squares indicate the two competing individuals. In this experiment, and contrary to that in [1], the vector population is not reversed at the end of the selection so that the propagation of the fittest individual is slowed down.

By using this iterative method for selection, the fittest individual will ultimately occupy the last slot of the vector population.

\section{B. Mutation}

In this experiment, a real-coded mutation operator is used as the main operator to tune the parameters of the evaluation function of each individual. We choose to use the polynomial mutation as devised in [11]. Its distribution parameter $\eta$ is set to 20. The mutated parameter $y$ is given below.

$$
y_{i}^{(1, t+1)}=x_{i}^{(1, t+1)}+\left(x_{i}^{(U)}-x_{i}^{(L)}\right) \vec{\delta}_{i}
$$

The parameter $\vec{\delta}_{i}$ is calculated from the polynomial probability distribution:

$$
\begin{gathered}
P(\delta)=0.5\left(\eta_{m}+1\right)\left(1-|\delta|^{\eta m}\right) \\
\bar{\delta}_{i}=\left\{\begin{array}{cc}
\left(2 r_{i}\right)^{1 /\left(\eta_{m}+1\right)}-1, & \text { If } \mathrm{r}_{\mathrm{i}}<0.5 \\
1-\left[2\left(1-r_{i}\right)\right]^{1 /\left(\eta_{m}+1\right)}, & \text { If } \mathrm{r}_{\mathrm{i}}>=0.5
\end{array}\right.
\end{gathered}
$$

Where:

$$
\begin{aligned}
& y_{i}^{(1, t+1)}=\text { new parameter after mutation } \\
& x_{i}^{(1, t+1)}=\text { parameter before mutation } \\
& x_{i}^{(U)}=\text { parameter upper boundary }
\end{aligned}
$$




$$
\begin{aligned}
& x_{i}^{(L)}=\text { parameter lower boundary } \\
& \eta=\text { probability to mutate the parameter close to original } \\
& \quad \text { parameter } \\
& r=\text { random number in }\left[\begin{array}{ll}
0 & 1
\end{array}\right] .
\end{aligned}
$$

Recall that each individual consists of six parameters denoting the weights of the different chess pieces (except the king and the pawn), double pawn and mobility. For each parameter, a specific value of its probability of mutation is assigned to it. For instance, the mutation probabilities for queen, rook, bishop, and knight parameters were all set to $25 \%$, while the mutation probabilities for a double pawn and mobility parameters were set to $10 \%$. Note that the mutation probabilities are relatively higher than the usual. This is to make sure that at least one parameter is mutated so that there will be no duplicated individuals in the population.

\section{Dy namic Boundary Strategy}

A good search method must promote diversity so that it is not trapped in a local optima. The search space in this application is very large. The diversity of a search method can be reflected by the standard deviations of each of the parameters. If the standard deviation is large then the diversity of the search method will be also large, whereas if the standard deviation is reduced, the diversity will also be reduced.

To solve the problem of converging to a local optima, we propose a new approach called Dynamic Boundary Strategy. This strategy (at the same time) maintains and controls the diversity of the search throughout the generations. In other approaches [1], it is required to fix the upper and lower boundaries for each of the individual's parameters.

The basic idea of the dynamic boundary strategy is to have an adjustable boundary for each parameter. Consider the following individual with the initial parameters:

\begin{tabular}{c|c|c|c|c|c} 
Queen & Rook & Bishop & Knight & Double Pawn & Mobility \\
\hline 9 & 5 & 3 & 3 & 0.5 & 0.1
\end{tabular}

The boundaries for the domain of each parameter are dynamic, however the diameter of that domain is constant. For instance, the diameters of the domains of the queen, the rook, the bishop, and the knight are all set to the value 2 . The double pawn's domain is set the value 0.2. The Mobility' boundaries are fixed however (upper boundary $=0.5$, lower boundary $=0.01$ ). For instance, if the weight of the queen is 9 , then its upper boundary is 11 and its lower boundary is 7 . If the weight of double pawn is 0.5 , then its upper and lower boundaries are respectively 0.7 and 0.3 .

For example, the parameters of the above individual after applying the polynomial mutation could be as the following:

\begin{tabular}{c|c|c|c|c|c} 
Queen & Rook & Bishop & Knight & Double Pawn & Mobility \\
\hline 8.25 & 5 & 3 & 3 & 0.5 & 0.1
\end{tabular}

Notice that the weight of the queen has been mutated from 9 to 8.25 . Then, the upper boundary of the queen will change to 10.25 while its lower boundary will become 6.25 . This means that when the next mutation occurs on the queen's weight, the possible weight for the queen will range from 10.25 to 6.25 .

In order to speed up the learning process, the weight of the queen can be forced to be at least equal to the highest parameter of the individual (such as rook, bishop, or knight). This is because it is sensible to think that the weight of the queen must be higher than the weight of any other chess piece (other than the king) as it is the most useful piece in a chess game. For example, consider the following values of the parameters of a given individual:

\begin{tabular}{c|c|c|c|c|c} 
Queen & Rook & Bishop & Knight & Double Pawn & Mobility \\
\hline 4.5 & 5 & 3 & 3 & 0.5 & 0.1
\end{tabular}

Notice that the Rook's weight is the highest. It is obvious that the Queen's weight should be at least as higher as that of the Rook. Therefore, we can bring the Queen's weight up to 5, which is now equal to the Rook's weight.

\begin{tabular}{c|c|c|c|c|c} 
Queen & Rook & Bishop & Knight & Double Pawn & Mobility \\
\hline 5 & 5 & 3 & 3 & 0.5 & 0.1
\end{tabular}

The main advantage of dynamic boundary strategy is that the search space in one generation of learning is much smaller compared to that of other fixed boundary strategies.

Indeed, the dynamic boundary strategy explores small portions of the search space at each generation. However, in the long run, it is still capable of exploring a huge part of the search space. This is due to its moving boundaries. The main idea is that at each generation, the search process is concentrated in one part of the search space.

Without this strategy, one needs to set good boundaries for the parameter's domain, otherwise, a sufficiently large domain must be thought of, which is not a trivial task. This is not required when using the dynamic boundary strategy

\section{EXPERIMENTAL RESULTS}

\section{A. Experimental Design}

A small population of five individuals is used for breeding a good solution. The values for each of the parameters were inserted manually to make sure that the initial population only consists of poor individuals (bad evaluation functions). The purpose was to see whether the dynamic boundary strategy would be able to ameliorate the fitness (evaluation function) of the individuals. The initial individuals of the population are shown in Table I. 
TABLE I. THE INDIVIDUALS OF THE INITIAL POPULATION

\begin{tabular}{|l|c|c|c|c|c|c|}
\hline & Queen & Rook & Bishop & Knight & $\begin{array}{c}\text { Double } \\
\text { Pawn }\end{array}$ & Mobility \\
\hline Individual 1 & 3 & 3 & 3 & 3 & 0.5 & 0.1 \\
\hline Individual 2 & 2.5 & 2.5 & 2.5 & 2.5 & 0.5 & 0.1 \\
\hline Individual 3 & 2 & 2 & 2 & 2 & 0.5 & 0.1 \\
\hline Individual 4 & 1.5 & 1.5 & 1.5 & 1.5 & 0.5 & 0.1 \\
\hline Individual 5 & 1 & 1 & 1 & 1 & 0.5 & 0.1 \\
\hline
\end{tabular}

Recall from the section II that our chess program was designed to search 3 ply and we can extend the search another 3 ply when the quiescence check operation is triggered. We run the program for 520 generations. The means and the standard deviations of the initial population are given below in Table II.

\section{B. Results}

After learning for 520 generations, the values of the parameters of the individuals have changed a lot. Figure 2 and Figure 3 respectively show the averages and the standard deviations of the parameters throughout the whole learning procedure (520 generations).

The averages of the weights and their standard deviations at the end of the experiments are given in Table III.

It is worth noting that in evolutionary algorithms that use a fixed boundary approach, the diversity of the search decreases gradually after many generations. Notice also that the standard deviations of the last population (using our strategy) shown in Table III have not changed too far from their initial standard deviations counterpart (shown in Table II).

\section{Success}

The weights of the Shannon's evaluation function [5] are as follows:

\begin{tabular}{c|c|c|c|c|c} 
Queen & Rook & Bishop & Knight & Double Pawn & Mobility \\
\hline 9 & 5 & 3 & 3 & 0.5 & 0.1
\end{tabular}

After running our chess program for 520 generations, the learning process produced the fittest individual whose parameter values are as follows

\begin{tabular}{c|c|c|c|c|c} 
Queen & Rook & Bishop & Knight & Double Pawn & Mobility \\
\hline 7.57 & 4.66 & 4.41 & 3.16 & 0.44 & 0.11
\end{tabular}

TABLE II. AVERAGES AND THE STDEVS AT THE INITIAL POPULATION

\begin{tabular}{|c|c|c|c|c|c|c|}
\hline & Queen & Rook & Bishop & Knight & $\begin{array}{c}\text { Double } \\
\text { Pawn }\end{array}$ & Mobility \\
\hline Average & 2 & 2 & 2 & 2 & 0.5 & 0.1 \\
\hline STDEV & 0.71 & 0.71 & 0.71 & 0.71 & 0.0 & 0.0 \\
\hline
\end{tabular}

TABLE III. AVERAGES AND STDEVS AT THE LAST POPULATION.

\begin{tabular}{|c|c|c|c|c|c|c|}
\hline & Queen & Rook & Bishop & Knight & $\begin{array}{c}\text { Double } \\
\text { Pawn }\end{array}$ & Mobility \\
\hline Average & 7.60 & 3.86 & 3.56 & 2.95 & 0.48 & 0.10 \\
\hline STDEV & 0.08 & 0.68 & 0.69 & 0.12 & 0.03 & 0.01 \\
\hline
\end{tabular}

This fittest individual was tested against a chess program that uses the Shannon's evaluation function. The results are shown in Table IV.

In the first game, the chess program based on the Shannon's evaluation function dominated the game, but the game ended with a draw because both players have reached the 50 move limit. However, in the second game, the fittest individual of our chess program took control of the game and ended it in just 42 moves ( 21 moves for each player). This result has shown that the fittest individual is strong enough to win against a wellestablished evaluation function.

We further tested our chess program based on the dynamic boundary strategy against a well-known commercial chess program called "Chessmaster 8000". The Chessmaster 8000 level of difficulty was set to players rating 1800 , which falls in the A class in the USCF rating. The fittest individual of our chess program played as White player for two games and the results are shown in Table $\mathrm{V}$.

In the first game, the Chessmaster 8000 beat the fittest individual in 81 moves. We consider this as a promising result as our fittest individual was competitive enough to last this long.

The second game ended with a draw because of the 50 move limit. However, our fittest individual did very well in this round as it was dominating the game, and had the game been extended, it would have beaten the ChessMaster 8000 . Our chess program was left with a rook and a pawn, while the Chessmaster 8000 was left with 2 pawns only.

\section{CONCLUSION}

In this paper, we have proposed the use a novel approach for the evaluation function of a chess playing game. This is called Dynamic Boundary Strategy

This method is quite effective because those parts of the search space that are not promising would not be visited. However, it is important to make sure that the diameter of the interval (distance between upper and lower boundary) of the weight of a given parameter must be large enough so that the

TABLE IV. FITTEST INDIVIDUAL AGAINST SHANNON’ EVALUATION FUNCTION.

\begin{tabular}{|l|c|c|c|}
\hline & Play as & Moves & Result \\
\hline Fittest Individual & White & 100 moves & Draw \\
\hline Fittest Individual & Black & 42 moves & Win \\
\hline
\end{tabular}




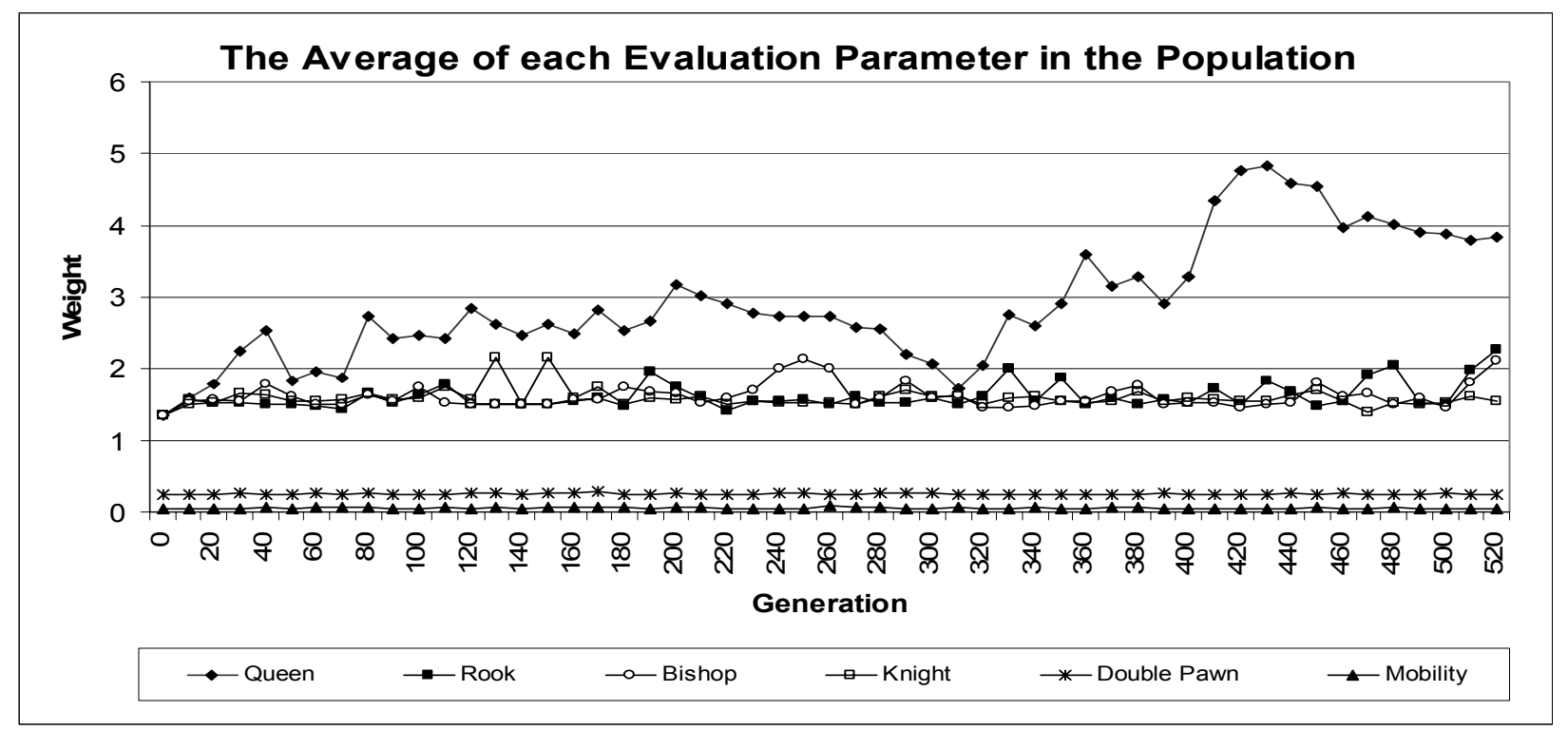

Figure 2. . Averages of each parameter throughout the learning process

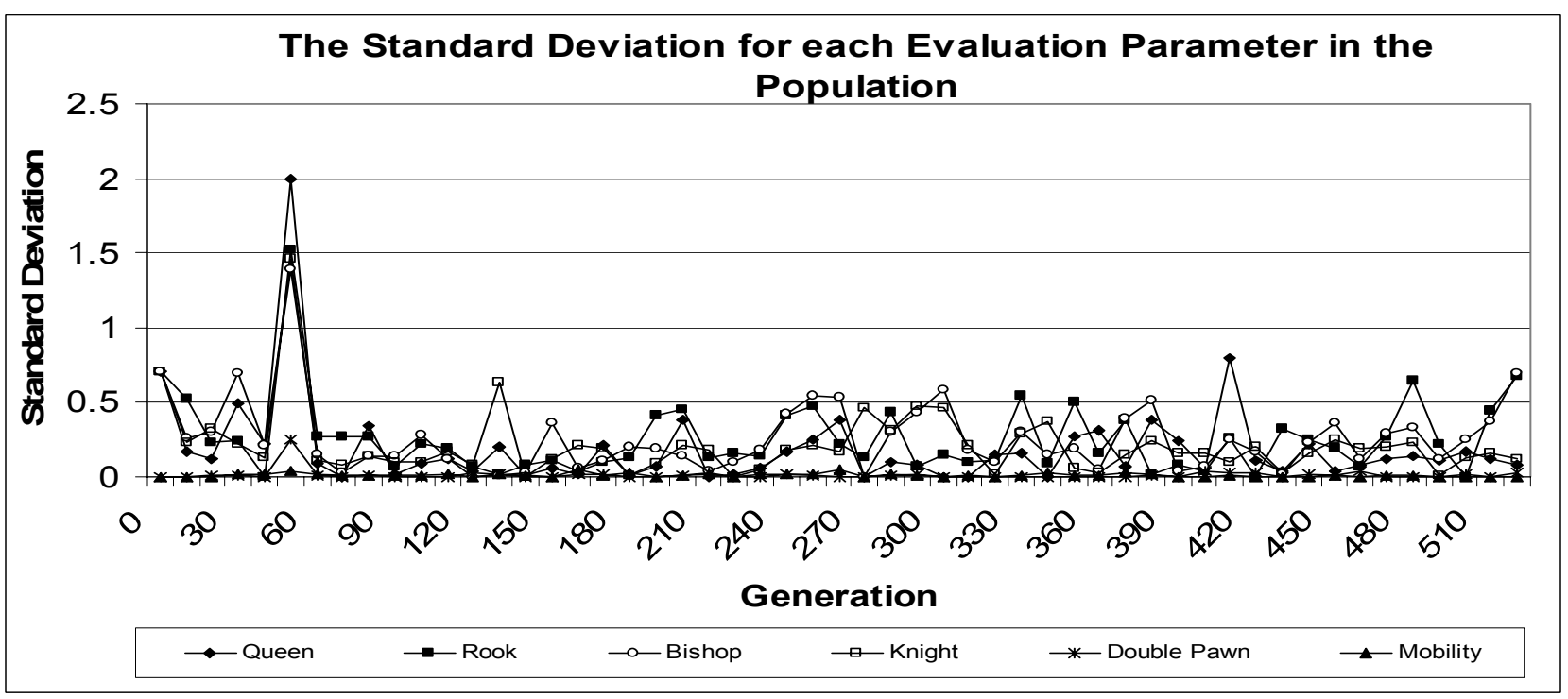

Figure 3. Standard deviations of each parameter throughout the learning process

search is not trapped into a local optima.

The fittest individual produced by our method is still unable to compete against a very good chess player (Chessmaster 8000), but it has proven that the learning algorithm was able to optimise (to some extent) the evaluation function that is used to determine a good move. It has beaten the chess program that uses Shannon's evaluation function.

In our future work, we will extend the search depth, to incorporate heuristics, and use a more complex evaluation function. This, we hope, will produce a very competitive chess playing individual.

TABLE V. FitTEST INDIVIDUAL AGAINST CHESSMASTER 8000.

\begin{tabular}{|l|c|c|c|}
\hline & Play as & Moves & Result \\
\hline Fittest Individual & White & 81 moves & Lost \\
\hline Fittest Individual & White & 100 moves & Draw \\
\hline
\end{tabular}


APPENDIX

$1^{\text {st }}$ game: Fittest Individual (White) Vs. Chessmaster 8000 (Black)

\begin{tabular}{|c|c|c|c|c|c|}
\hline 1 & $\mathrm{e} 2 \mathrm{e} 3$ & g8f6 & 26 & $\mathrm{a} 1 \mathrm{a} 4$ & c8f5 \\
\hline 2 & $\mathrm{fld} 3$ & $\mathrm{~d} 7 \mathrm{~d} 6$ & 27 & h1b1 & b7b5 \\
\hline 3 & $\mathrm{~d} 1 \mathrm{f} 3$ & h8g8 & 28 & b1b5 & f5h3 \\
\hline 4 & $\mathrm{~b} 2 \mathrm{~b} 3$ & $\mathrm{~b} 8 \mathrm{~d} 7$ & 29 & $\mathrm{~g} 2 \mathrm{~h} 3$ & e7f6 \\
\hline 5 & b1c3 & h7h5 & 30 & c3f6 & a6b5 \\
\hline 6 & f3f5 & a7a6 & 31 & $\mathrm{a} 4 \mathrm{a} 8$ & e8d7 \\
\hline 7 & g1f3 & $\mathrm{c} 7 \mathrm{c} 5$ & 32 & h3h4 & $\mathrm{d} 7 \mathrm{e} 6$ \\
\hline 8 & $\mathrm{~h} 2 \mathrm{~h} 3$ & e7e6 & 33 & $\mathrm{a} 8 \mathrm{c} 8$ & e6f6 \\
\hline 9 & $\mathrm{f5f4}$ & $\mathrm{f} 6 \mathrm{~d} 5$ & 34 & h4h5 & f6e 6 \\
\hline 10 & $\mathrm{c} 3 \mathrm{~d} 5$ & e6d5 & 35 & h5h4 & e6e7 \\
\hline 11 & f4f5 & $\mathrm{d} 8 \mathrm{c} 7$ & 36 & $\mathrm{e} 3 \mathrm{e} 4$ & $\mathrm{~b} 5 \mathrm{~b} 4$ \\
\hline 12 & c1b2 & d7f6 & 37 & c8c7 & e7f6 \\
\hline 13 & $\mathrm{f} 5 \mathrm{f} 4$ & f6e4 & 38 & h4g3 & f6e5 \\
\hline 14 & $\mathrm{a} 2 \mathrm{a} 4$ & g7g5 & 39 & c7e7 & $\mathrm{e} 5 \mathrm{~d} 4$ \\
\hline 15 & $\mathrm{f} 3 \mathrm{~g} 5$ & g8g5 & 40 & g3g2 & b4b3 \\
\hline 16 & $\mathrm{f} 2 \mathrm{f} 3$ & c7a5 & 41 & g2g3 & b3b2 \\
\hline 17 & ald1 & g5g2 & 42 & $\mathrm{e} 7 \mathrm{~b} 7$ & $\mathrm{~d} 4 \mathrm{e} 4$ \\
\hline 18 & f3e4 & $\mathrm{c} 5 \mathrm{c} 4$ & 43 & $\mathrm{~b} 7 \mathrm{~b} 2$ & $\mathrm{f} 7 \mathrm{f5}$ \\
\hline 19 & elfl & f8e 7 & 44 & $\mathrm{~b} 2 \mathrm{~b} 4$ & $\mathrm{e} 4 \mathrm{~d} 5$ \\
\hline 20 & flg2 & $\mathrm{c} 4 \mathrm{~d} 3$ & 45 & g3g2 & $\mathrm{f} 5 \mathrm{f} 4$ \\
\hline 21 & b3b4 & $\mathrm{a} 5 \mathrm{~b} 4$ & 46 & $\mathrm{~b} 4 \mathrm{f} 4$ & $\mathrm{~d} 5 \mathrm{e} 5$ \\
\hline 22 & $\mathrm{~b} 2 \mathrm{c} 3$ & $\mathrm{~b} 4 \mathrm{a} 4$ & 47 & $\mathrm{f} 4 \mathrm{f} 7$ & $\mathrm{e} 5 \mathrm{~d} 5$ \\
\hline 23 & d1a1 & a4e4 & 48 & $\mathrm{f} 7 \mathrm{f6}$ & $\mathrm{d} 5 \mathrm{c} 5$ \\
\hline 24 & $\mathrm{f} 4 \mathrm{e} 4$ & $\mathrm{~d} 5 \mathrm{e} 4$ & 49 & f6f7 & $\mathrm{c} 5 \mathrm{~d} 5$ \\
\hline 25 & $\mathrm{c} 2 \mathrm{~d} 3$ & $\mathrm{e} 4 \mathrm{~d} 3$ & 50 & $\mathrm{~g} 2 \mathrm{~g} 3$ & $\mathrm{~d} 5 \mathrm{c} 5$ \\
\hline
\end{tabular}

$2^{\text {nd }}$ game: Fittest Individual (White) Vs. Chessmaster 8000 (Black)

\begin{tabular}{|r|ll|l|ll|}
\hline 1 & $\mathrm{e} 2 \mathrm{e} 3$ & $\mathrm{~g} 8 \mathrm{f} 6$ & 26 & $\mathrm{~d} 2 \mathrm{e} 3$ & $\mathrm{f} 4 \mathrm{~g} 5$ \\
2 & $\mathrm{f} 1 \mathrm{~d} 3$ & $\mathrm{~d} 7 \mathrm{~d} 6$ & 22 & $\mathrm{~h} 1 \mathrm{~g} 1$ & $\mathrm{~d} 8 \mathrm{~h} 4$ \\
3 & $\mathrm{~d} 1 \mathrm{f3}$ & $\mathrm{h} 8 \mathrm{~g} 8$ & 23 & $\mathrm{~g} 1 \mathrm{~h} 1$ & $\mathrm{~d} 5 \mathrm{e} 4$ \\
4 & $\mathrm{~b} 2 \mathrm{~b} 3$ & $\mathrm{~b} 8 \mathrm{~d} 7$ & 24 & $\mathrm{~d} 3 \mathrm{c} 4$ & $\mathrm{e} 4 \mathrm{e} 3$ \\
5 & $\mathrm{~b} 1 \mathrm{c} 3$ & $\mathrm{~h} 7 \mathrm{~h} 5$ & 25 & $\mathrm{~h} 1 \mathrm{~h} 2$ & $\mathrm{~h} 4 \mathrm{f} 4$ \\
6 & $\mathrm{f} 3 \mathrm{f} 5$ & $\mathrm{a} 7 \mathrm{a} 6$ & 27 & $\mathrm{~g} 2 \mathrm{~h} 1$ & $\mathrm{~g} 5 \mathrm{e} 7$ \\
7 & $\mathrm{~g} 1 \mathrm{f} 3$ & $\mathrm{c} 7 \mathrm{c} 5$ & 28 & $\mathrm{~h} 1 \mathrm{~g} 2$ & $\mathrm{e} 7 \mathrm{e} 4$ \\
8 & $\mathrm{~h} 2 \mathrm{~h} 3$ & $\mathrm{e} 7 \mathrm{e} 6$ & 29 & $\mathrm{~g} 2 \mathrm{f} 1$ & $\mathrm{c} 8 \mathrm{e} 6$ \\
9 & $\mathrm{f} 5 \mathrm{f} 4$ & $\mathrm{f} 6 \mathrm{~d} 5$ & 30 & $\mathrm{c} 4 \mathrm{e} 2$ & $\mathrm{e} 4 \mathrm{e} 3$ \\
10 & $\mathrm{c} 3 \mathrm{~d} 5$ & $\mathrm{e} 6 \mathrm{~d} 5$ & 31 & $\mathrm{~b} 2 \mathrm{c} 1$ & $\mathrm{e} 6 \mathrm{~h} 3$ \\
11 & $\mathrm{f} 4 \mathrm{f} 5$ & $\mathrm{~d} 8 \mathrm{c} 7$ & 32 & $\mathrm{~h} 2 \mathrm{~h} 3$ & $\mathrm{e} 3 \mathrm{~h} 3$ \\
12 & $\mathrm{c} 1 \mathrm{~b} 2$ & $\mathrm{~d} 7 \mathrm{f} 6$ & 33 & $\mathrm{flf} 2$ & $\mathrm{~h} 3 \mathrm{~h} 4$ \\
\hline
\end{tabular}

\begin{tabular}{|l|ll|l|ll|}
\hline 13 & f5f4 & f6e4 & 34 & f2f1 & e8c8 \\
14 & a2a4 & g7g5 & 35 & c1e3 & h4f6 \\
15 & f3g5 & g8g5 & 36 & f1g2 & f6a1 \\
16 & f2f3 & g5g2 & 37 & e2h5 & d8g8 \\
17 & f3e4 & c7b8 & 38 & g2f3 & a1f1 \\
18 & a4a5 & b8c7 & 39 & f3e4 & g8e8 \\
19 & e1f1 & f8h6 & 40 & e4d5 & e8e5 \\
20 & f1g2 & h6f4 & 41 & d5d6 & \\
21 & e3f4 & c7d8 & & & \\
\hline
\end{tabular}

\section{ACKNOWLEDGMENT}

The author Hendra Suhanto Poh thanks Nasa Tjan for his assistance in developing the chess engine, and Gunawan for providing a facility to run the experiments.

\section{REFERENCES}

[1] G. Kendall and G. Whitwell, "An evolutionary approach for the tuning of a chess evaluation function using population dynamics," Proceedings of the 2001 Congress on Evolutionary Computation, vol. 2, pp. 9951002, May 2001.

[2] G. Whitwell, "Artificial Intelligence Chess with Adaptive Learning," Bachelor Degree Dissertation Thesis, the University of Nottingham, May 2000.

[3] D. B. Fogel, T. J. Hays, S. L. Hahn, and J. Quon, "A self-learning evolutionary chess program," Proceeding of the IEEE, vol. 92, Issue 12, pp. 1947-1954, Dec. 2004.

[4] D. Kopec, M. Newborn, and M. Valvo, "The 22d annual ACM international computer chess championship," Communication of the ACM, vol. 35, Issue 11, pp. 100-110, Nov 1992.

[5] E. Shannon, Claude, "XXII. Programming a Computer for Playing Chess," Philosophical Magazine, Ser. 7, vol. 41, No. 314, Mar 1950.

[6] Z. Michalewicz, "Genetic algorithms + data structures = evolution programs / Zbigniew Michalewicz," Berlin: Springer-Verlag, $3^{\text {rd }}$ Edition revised and extended, 1996.

[7] S. J. Russel and P. Norvig, "Artificial Intelligence: A Modern Approach," Upper Saddle River, N.J.: Prentice Hall/Pearson Education, $2^{\text {nd }}$ Edition, 2003.

[8] D. Goldberg, "Genetic Algorithms in Search, Optimization, and Machine Learning/ David E. Goldberg," Boston, Mass.: Addison, Wesley, 1989.

[9] H. Kaindl, "Tree searching algorithms," in Computers, Chess, and Cognition, T. A. Marsland and J. Schaeffer, Eds. New York: SpringerVerlag, pp. 133-168, 1990.

[10] F. G. Luger, "Artificial Intelligence: Structures and Strategies for Complex Problem Solving," Addison Wesley Publishing Company, $4^{\text {th }}$ Edition, p. $144-152,1997$.

[11] K. Deb and K. B. Agrawal, "Simulated Binary Crossover For Continuous Search Space.” Complex System, vol 9, pp.115-148, 1994. 